\title{
Las nuevas tecnologías y los adolescentes en exclusión social: el proyecto Ciberándara.
}

\author{
Pilar Viadero Sánchez \\ pivisaltelefonica.net \\ Pedagoga y educadora social (Santander)
}

\section{Presentación}

La experiencia que a continuación se quiere compartir nace en el ámbito de la educación no formal, concretamente desde el trabajo socioeducativo con adolescentes y jóvenes en situación de exclusión social de la ciudad de Santander (Cantabria). Su desarrollo es fruto de las vivencias directas de un grupo de educadores que realizan su práctica educativa en este contexto, y de las reflexiones que estos años de trabajo han permitido que avancemos y nos formemos interiormente un poco más como profesionales de la educación y como personas. Esta intervención te ayuda a comprender la vida de estos chicos, pero más allá te enseña a conocer la vida en general, el estado de la sociedad, de las estructuras, de lo que dicen que es y no es, de lo que dicen que está y no está.

Esta experiencia tiene uno de sus puntos fuerte de trabajo educativo en el aspecto escolar, o más bien desescolar, ya que se interviene con chicos menores de 16 años (12-16) que se encuentran desescolarizados, que han agotado todos los recursos que el sistema educativo les puede ofrecer, han sido expulsados con expedientes disciplinarios, no se integraban en la clase, en el centro...., han tenido comportamientos en la calle que les han llevado al Juzgado de Menores.

En esta situación desde el Proyecto Ándara se tiene como objetivo el buscar nuevas formas de educación para estos chicos, y despertarles su interés por volver a la escuela, o al menos prepararlos para realizar otra formación cuando tengan la edad necesaria. La mayor parte de estos chicos nos llegan con expedientes de protección o reforma, o desde el Programa de Absentismo escolar, por lo que la coordinación con las instituciones y con la administración a través de los equipos educativos que poseen es cercana; cada uno de estos educadores tiene asignado el caso de un chico, para lo que establecemos la coordinación adecuada.

El Proyecto Ándara es un recurso socioeducativo para trabajar con estos chicos; su utopía de futuro es tender a desaparecer, pues el mayor deseo sería que el propio sistema escolar tuviera las medidas de atención a la diversidad necesarias, pero la realidad nos lleva a la situación que vivimos, y estos chicos requieren una atención muy individualizada y muy específica. Por eso este trabajo se basa en la continua innovación, reflexión-acción-reflexión; desde aquí se presenta el Proyecto Ándara y hace partícipe, a quien se interese, de algunas metodología educativas que están en proyección para poder hacer reales las medidas de atención a la diversidad dentro de la intervención con colectivos de exclusión social y escolar.

\section{Antecedentes del proyecto y contexto}

Desde Cáritas Diocesana de Santander lleva desarrollándose una labor de intervención con adolescencia y juventud en riesgo desde 1995, valorando la necesidad de comenzar desde esta institución a realizar un trabajo de intervención con estos chicos que comenzaban a manifestar comportamientos delictivos y a mantener un contacto con el Juzgado de Menores. Por la ausencia de un recurso específico se creó el Programa de Medidas en Medio abierto, mediante un acuerdo con el Gobierno de Cantabria. 
Desde este programa se empezó a trabajar con chicos que presentaban realidades personales y sociales difíciles, donde la intervención venía marcada por un primer comienzo obligatorio, y más aún vinculado a lo judicial. El equipo de educadoras en ese momento tenía claros sus principios pedagógicos, que no coincidían con la idea de sanción y castigo que un modelo penal y punitivo llevaba consigo, por lo que la meta a conseguir era pasar de ese conocimiento y encuentro obligatorio a una relación basada en la voluntariedad, y el establecimiento de un clima de confianza y empatía que permitiera hacer crecer procesos verdaderamente educativos y positivos para el desarrollo del chico/a.

Desde el primer momento hemos considerado que todo chico necesita adquirir una responsabilidad personal para poder interaccionar con el medio en el que vive; en este caso entraba en juego la responsabilidad penal; es difícil que un chico que ha nacido y crecido en un entorno vulnerable al riesgo, sin tener cubiertas unas necesidades básicas, desde la seguridad material hasta la afectiva y que no ha configurado un proceso de socialización basado en modelos positivos, pueda hacerse cargo y responder a una norma social y penal que le es ajena. No es capaz de responder ante su propia vida, menos aún lo va a hacer ante algo que no entiende y con lo que no se identifica. Esto hace que para poder llegar a un resultado esperanzador con estos chicos, partamos de criterios educativos y de procesos que incidan en lo más profundo de su realidad y necesidades, no quedándose en el simple castigo ante un hecho que la sociedad del bienestar ha catalogado como perjudicial para su seguridad.

Esto ha marcado una manera de ser y de hacer en el equipo educativo; se ha realizado un trabajo integral con los chicos que llegaban con una medida judicial que cumplir, atendiendo a su realidad, necesidades y posibilidades. El esfuerzo ha estado siempre presente, en un trabajo en el que los resultados son muy lentos cuando los hay y los fracasos se hacen muy visibles. Mucho tiempo dedicado a buscar soluciones para encaminar la vida de los chicos, pero muy pocos caminos de esperanza. La acción directa nos hacía pensar en valorar y analizar lo que hacíamos y lo que podríamos hacer por ir más allá, por llegar a la acción indirecta que nos guiara hacia un cambio a nivel estructural.

Es así como poco a poco vamos llegando a una reflexión sobre la forma de canalizar y aprovechar todo el esfuerzo que el equipo estaba dispuesto a realizar por estos chicos, y por cambiar un poquito el estado de las "cosas". La reflexión que se realiza es la siguiente, fruto de la experiencia y del trabajo realizado entre 1995 y 2000 ;

Ausencia de recursos adaptados a las necesidades que presentan, teniendo que ser ellos los que se adapten a lo que se les ofrece, cuando el proceso debiera ser a la inversa, no teniendo ellos hábitos y destrezas para hacer frente a esas exigencias.

- Carencia de hábitos, capacidad de asumir normas, habilidades relacionales, escasa tolerancia a la frustración, bajo nivel de conocimientos ante el fracaso en el medio escolar, desmotivación..., fruto todo ello de las situaciones ambientales que viven estos chicos en condiciones de una vida llena de riesgos.

Esto nos lleva a pensar en crear un recurso educativo para desarrollar la intervención con los chicos, y perseguir su promoción social como persona, al tiempo que tender a que ellos mismos sean capaces de desarrollar un proyecto de vida al margen de entornos vulnerables al riesgo. El objetivo es aprovechar el conocimiento previo que se ha logrado de estos chicos, de su realidad, de sus necesidades, de lo que hay y no hay en cuanto a recursos formativos y prelaborales. Mucho tiempo dedicado a buscar soluciones en recursos que no acababan de encajar con el perfil que ellos presentan: se les pide mucho que nadie primero les ha sabido transmitir y enseñar; no tienen hábitos de trabajo, capacidad de esfuerzo, motivación hacia el estudio, perspectivas de futuro; y si las tienen están encerradas en las costumbres familiares, en el terreno de la delincuencia, marginación y supervivencia.

Surge así un nuevo proyecto que nace de un trabajo previo dentro de la misma organización, lo cual se valora muy positivamente porque no nace sin sentido, sino que se ha ido haciendo poco a poco hasta conocer bien la necesidad: Proyecto Ándara. 
El Taller Ándara es un recurso socioeducativo que atiende a chicos que presentan unas situaciones de vida caracterizadas por un alto índice de riesgo, teniendo a su alrededor realidades sociales, familiares y personales que no permiten un adecuado desarrollo como ser individual y perteneciente a un grupo social; se hayan inmersos en procesos de fracaso y abandono escolar, familiar, comunitario, y se encuentran cada vez más cerca de la calle y la vida delictiva.

En el Taller atendemos a chicos de edades comprendidas entre los 13 y 16 años, pero la intervención realizada es más amplia, y ofrece un proceso de acompañamiento a los chicos que van terminando su paso por el taller, así como a otros que forman parte del grupo de iguales, que consideramos positiva como actuación indirecta en beneficio de los que pertenecen al proyecto; esto hace que la edad que atendemos se amplíe hasta los 18-20 años.

El Taller Ándara concreta su acción en dos actuaciones: la realizada con el grupo que participa en la dinámica diaria del Taller, y los chicos que cumplen de forma puntual alguna medida dictada por el Juzgado de Menores. Queremos ofrecer la posibilidad de trabajar educativamente lo que en un principio es judicial, fomentando la responsabilidad personal como base de la responsabilidad penal. De esta forma, otro de nuestros objetivos es el despertar motivaciones en los chicos que cumplen las medidas para realizar alguna actividad (los que están en mayor situación de riesgo y sin ocupación), incluso de incorporarse al trabajo educativo diario que llevamos en el taller con el grupo estable.

\section{Desarrollo del proyecto y contenidos}

El Taller Ándara parte de la educación por el trabajo para fomentar el desarrollo y aprendizaje de hábitos de trabajo (normas, horarios, limpieza, respeto, esfuerzo...), habilidades de comunicación, análisis de conductas, resolución positiva de conflictos, incitar e ilusionar por un proyecto de futuro en los chicos. A través de la jardinería y la huerta como elemento central nos planteamos la intervención grupal; no se trata de especializar a los chicos en un oficio sino utilizar lo que las relaciones en colectivo pueden aportar a los comportamientos de cada uno en grupo y consigo mismo (el trabajo como herramienta para el análisis y transformación de conductas y desarrollo de hábitos).

En un primer momento conocemos la realidad del chico que se nos plantea como posible participante del Taller, en contacto y coordinación con otros agentes educativos y sociales, los cuales están trabajando con el chico; si se valora por ambas partes que puede ser positivo para él, que se han agotado otros recursos normalizados (en caso de los menores de 16 años), y que el grupo de taller en ese momento puede incorporar a otro chico, se plantea a nivel formal el inicio en el Taller Ándara, manteniendo siempre la coordinación continua con la figura educativa o social de referencia.

Según la situación y necesidades de cada chico se elabora un Proyecto Educativo Individualizado, teniendo en cuenta tiempos, ritmos, estrategias de intervención, acción individual..., y comenzará así su participación en los diferentes contenidos del taller. El proceso de cada chico en el taller es diferente; aunque realicen actividades de grupo, hay un trabajo muy individualizado, ya que vamos a realizar una intervención integral que irá más allá del tiempo diario en el taller (tiempo libre, calle, amigos, familia, conductas individuales...).

La dinámica se desarrolla a través de diferentes contenidos, cada uno de los cuales tiene su correspondiente programación y objetivos; la actividad se realiza de lunes a viernes, y los contenidos concretos son; jardinería y huerta, aula formativa (espacio escolar con menores de 16 años), orientación laboral, vida cotidiana, deporte, actividades complementarias, salidas socioculturales. Las Asambleas son un elemento muy importante, con el que se pretende implicar a los chicos en el desarrollo del taller, hacerles partícipes y buscar su motivación e interés. Con los chicos menores de 16 años cobra mucha relevancia el componente escolar, ya que han abandonado ya el centro educativo (absentistas, expulsados con expediente disciplinarios...), y nuestro objetivo es coordinarnos con los centros y poder trabajar con ellos en el taller un currículo adaptado de forma individualizada; mantenemos una estrecha coordinación con el Programa de Absentismo. 
Una vez que se considera que se han cumplido los objetivos de la participación del chico en el Taller se realiza un acompañamiento individualizado con el fin de incorporarle en un recurso prelaboral o laboral, según las posibilidades y la situación del chico. Siempre hay una coordinación continua con las figuras de referencia desde las que llega el chico/a al taller, para realizar una actuación integral, que abarque todo el contexto global de la vida del chico.

En este contexto de intervención educativa es importante la creatividad en las formas de hacer la labor pedagógica, al ser chicos que llegan con una fuerte desmotivación hacia todo aprendizaje. Como metodología ha resultado muy favorable la educación por el trabajo, con la que a través de una huerta y las relaciones que la producción establece, se consiguen desarrollar en los chicos hábitos de trabajo, normas, responsabilidades, reparto de funciones, labor de equipo..., al tiempo que se introducen conceptos académicos básicos (áreas instrumentales del currículo) desde un aprendizaje constructivo y por descubrimiento.

Pero no cesa ahí la búsqueda de nuevas metodologías y formas de hacer. Es aquí donde entran en juego en este proyecto las nuevas tecnologías, desde la reflexión que la práctica ha permitido, así como del conocimiento de los chicos y sus centros de interés.

\section{El proyecto Ciberándara}

Así aparece en el Taller Ándara una nueva herramienta de trabajo: Ciberándara. Esta herramienta es aún un proyecto sin desarrollar; se han diseñado sus objetivos y contenidos como metodología de trabajo en el taller Ándara. A continuación se anotan algunas de las señas más significativas de este nuevo espacio que se pretende crear para trabajar con estos chicos.

Ciberándara es un nuevo espacio educativo para trabajar el elemento "aula" con los chicos que participan en el taller, aunque también en él se podrán encontrar posibilidades que sean útiles para el desarrollo de los otros contenidos del proyecto: jardinería y huerta, talleres y actividades complementarios, orientación formativo-laboral...

Es un proyecto de trabajo que busca adaptarse a la realidad de los chicos, partir de sus intereses y para ello busca estímulos lo suficientemente atractivos como para ganar su atención y motivarles. La situación escolar en el medio formal que han vivido no ha resultado muy exitosa, creando un prejuicio hacia la parte formativa-educativa que Ándara trae consigo. Esto ha generado que se piense y reflexione sobre nuevas formas de aprendizaje, con el objetivo de acercarles a la cultura y a las herramientas de trabajo que les van a poder ser útiles en un futuro inmediato.

Ciberándara plantea la construcción de un nuevo escenario educativo, el ciberespacio, y en lugar de adaptar el aula a la nueva realidad que la sociedad de la información trae consigo e introducir en la clase los cambios necesarios, pretende dejar el aula convencional y salir en busca de nuevos espacios físicos (abrir las puertas de la educación a la sociedad, a la vida), que los chicos tengan que trasladarse a otro lugar, a aquel que ellos conocen y les interesa, para partir desde el primer momento de una motivación previa, y buscar todas las fórmulas que estimulen su creatividad y llamen su atención.

Este planteamiento pedagógico también se basa en la actitud de los chicos respecto a las tecnologías, hacia las que muestran un interés significativo. Se ha trabajado con ellos un cuestionario con el fin de obtener información sobre sus gustos y conocimientos en el manejo del ordenador y de la red.

Un nuevo centro de interés ha surgido en los últimos años que atrae e interesa a los chicos, el ciberespacio, y con él han aparecido locales que ofrecen el servicio de la red, con los distintos usos que se derivan de ella, los conocidos "ciber". Los chicos conocen estos lugares y los frecuentan, se sientes atraídos, encuentran en ellos ocio y diversión: dominan los usos de la red casi mejor que los adultos (aunque se limiten a determinados elementos; juegos, chats...), y muestran que tienen capacidades que mediante una metodología tradicional no salían a la luz. 
Esta nueva forma de hacer pedagógico que aúna la educación, las nuevas tecnologías y la exclusión social plantea un estilo de educación determinada, que rompe esquemas predeterminados de antemano. La educación debe abrir los ojos al presente y al futuro, romper esquemas rígidos y apostar por un trabajo global e integrado, que parta de la realidad y enseñe a vivir y convivir con ella y en ella.

En el aula Ándara se ha trabajado con fichas didácticas en las que se derramaban los contenidos de forma globalizadora, a partir de temas de interés para los chicos, pero las actividades y la metodología han estado muy limitadas y condicionadas al tiempo que podíamos dedicar, al tener muchas otras funciones a nuestro cargo.

Ciberándara plantea una idea novedosa, fruto de las reflexiones realizadas en torno a la valoración del tiempo previo de experiencia y trabajo; es necesario romper esquemas rígidos de la educación tradicional, salir del aula como espacio educativo convencional y utilizar los nuevos espacios que la sociedad de la información y la comunicación nos presenta. El planteamiento es el de introducir en el proceso de enseñanza-aprendizaje de los chicos participantes en Ándara las nuevas tecnologías, pero en lugar de entrar ellas en nuestro lugar de actuación, vamos a salir nosotros a su encuentro.

Al tiempo de ofrecerles una nueva forma de aprender, se pretende fomentar nuevos usos de su tiempo libre, ya que éste está continuamente lleno de riesgos; además, son chicos que pasan mucho tiempo en la calle, con elevados niveles de consumo de drogas y otras conductas problemáticas e incluso delictivas.

La propuesta metodológica que se quiere introducir en el Taller Ándara es la de aprovechar esos espacios lúdicos e introducir en ellos el proceso de enseñanza-aprendizaje que la vida misma y la realidad de los chicos nos va mostrando. Es muy importante partir de la realidad y de las necesidades presentes y futuras, sin agotar la atención de los chicos con contenidos en los que no encuentren una finalidad; por su situación necesitan un aprendizaje que les prepare para la vida y para saber enfrentarse y desenvolverse en los que vaya aconteciendo.

En principio se está realizando una propuesta general orientativa de las líneas a perseguir con el desarrollo de este proyecto, que de continuar adelante se especificarían e irían adaptándose a las diferentes etapas del taller, teniendo en cuenta su cambiante realidad, y los distintos chicos que pueden pasar a lo largo del curso.

\subsection{Actividades de Ciberándara}

Las actividades irán encaminadas al aprendizaje del uso del ordenador y manejo de la red, y a relacionar la adquisición de contenidos de distintas áreas temáticas a través del uso de los medios informáticos e Internet.

Entre ellas podría destacar:

- Aprendizaje del manejo del ordenador, conocimientos informáticos.

- Utilizar lo aprendido en aquello que desde el proyecto necesitemos; datos relacionados con la producción en la huerta, inventario de materiales, redacción de escritos, rellenar documentos...

\section{- Aprender a usar Internet}

- Preparar los contenidos y temas formativos partiendo de intereses de los chicos y tener la red como almacén de información que hay que aprender a utilizar de forma crítica y constructiva.

- Complementar el uso de los medios informáticos con audiovisuales, fomentar la relación del grupo a través de actividades que favorezcan este trabajo. 
- Trabajar el área de instrumentales básicas.

- Complementar el taller de vida cotidiana; búsqueda en Internet de documentos de interés, callejeros de la ciudad, información sobre determinados temas, resolución de gestiones...

- Orientación laboral; búsqueda de empleo, contactos a través de correo electrónico...

- Programas informáticos educativos (CD-Rom Mundo gitano; actividades, historia, juegos, fotos, música, costumbres, actualidad...)

Comunicarse a través de la red con otras personas; colaboradores del proyecto, compañeros, compradores de los productos de la huerta...

- Conocer otras experiencias de trabajo con adolescentes, contactar y comunicarse a través de la red.

La propuesta vendría a poner en coordinación el Proyecto Ándara con estos espacios, los ciber; por las mañanas es un espacio que no suele tener mucho uso, ya que por lo general los chicos los frecuentan por las tardes, por lo que el planteamiento vendría a involucrar uno de estos locales con nuestro proyecto, estudiando las vías para poder hacerlo de forma realista, pensando también en el interés y beneficio del propietario del ciberlocal. De esta forma y a través de esta propuesta, nosotros podríamos trabajar con los chicos en su propio entorno, desde un recurso por el que se sienten atraídos, y el propietario podría ver funcionar su local en horario de mañana.

\subsection{Valoraciones desde la experiencia}

Como reflexión desde mi práctica como educadora en el Proyecto Ándara quiero compartir algunas inquietudes y reflexiones. Con estas ideas, iniciativas, sueños, locuras, vidas, experiencias... quiero compartir mi día a día y el de los chicos con los que trabajo con todo aquel que esté en este bonito mundo de la educación y de la exclusión social, y acercar algo tan actual y necesario como son las nuevas tecnologías a nuestra forma de trabajar y de llegar a los chavales.

La práctica diaria con ellos hace que tengamos que estar continuamente innovando, reflexionando, buscando soluciones, metodologías..., y es importante hacerlo desde el prisma de su realidad y de su historia. De nada nos va a servir repetir fórmulas escolares, esquemas elaborados pensando en chicos con unas necesidades cubiertas, con una estructura de vida, con unas seguridades satisfechas, con ilusiones, con adultos de referencia, con posibilidades reales y visibles.

En ocasiones puede parecer que las "brillantes ideas" de los educadores que trabajan desde ámbitos no formales o informales no llegarán más que a ser sueños y utopías, pero nacen de la más sincera y atenta mirada a los ojos de los chicos, a su mundo; por eso a veces parecerán locuras. Pero la vida necesita un poquito de esa locura sana que nos hace ilusionarnos y disfrutar trabajando por defender los derechos que estos chicos tienen a recibir educación, cultura y formación. Tal vez proyectos como este suenen a disparate para aquellos que conciben la educación bajo el hermetismo y estatismo de fórmulas magistrales, pero los susodichos posiblemente conozcan poquito de cómo son, viven, sienten y se relacionan estos chicos.

La calle ha sido su principal escuela, por lo que habrá que utilizar ésta y los elementos más cercanos a su realidad para educarles, porque es donde están y van a estar, porque tal vez no haya un padre o una madre esperando en casa para darle un beso o una sonrisa al llegar del "cole", porque tal vez nadie les diga si tienen o no tienen que ir a aprender y a formar su futuro.

Ellos me hacen aprender mucho, y me ayudan a elaborar mi teoría pedagógica; con ellos he podido darme cuenta de que tengo que estar abierta a todas las posibilidades que me brinden un poquito de esperanza. De ahí esta propuesta, este intento de llegar a sus barrios, a sus espacios, a sus intereses, y darles un toque de educación, de aprendizaje, de diversión. Nuestra escuela estará en todos aquellos 
rincones donde se pueda educar, donde estén los chavales, donde se sientan a gusto, donde nos den la oportunidad de ayudarles y guiarles en su crecimiento como seres individuales y sociales.

Tenemos un papel muy importante con estos chicos como figuras de referencia y de enseñanza, así como de orientación y ayuda en su transición a la vida adulta. Tanto esta experiencia como cualquier otra que se desarrolle para estimular los procesos de enseñanza-aprendizaje en contextos de exclusión social, han de tender a un cambio global; detrás de las acciones concretas debe haber una meta más amplia, más integral, en busca de un cambio en las estructuras socio-políticas.

Los entornos a los que se hace referencia a lo largo de esta exposición (exclusión y marginación social, o bien zonas vulnerables a pasar a esa situación) están comprados por la economía de mercado y sumisos al consumo indiscriminado. La falta de cultura y de desarrollo del pensamiento crítico y otras capacidades que la educación debiera transmitir a las personas, hace que estén sin defensas ante la oferta atractiva que les lanzan incesantemente; no ofrecen oposición a ella, los escasos recursos que tienen se esfuman en cuanto llegan al bolsillo. Los chicos con los que trabajo están también rendidos ante el mundo de los nuevos sistemas tecnológicos; el teléfono móvil, la playstation, el vídeo o dvd...., todos ellos están a la orden del día.

La sociedad de consumo ha elegido víctimas fáciles que no van a reflexionar sobre su propia injusticia, tienen en este sector de la sociedad la más fácil presa de sus campañas de marketing, donde todo parece importante para solucionar la vida, y donde esas familias y esos chicos encuentran un modo de igualarse al resto de la sociedad, a otros que sí pueden acceder a ello, a otros que gozan de una situación económica favorable a la posesión de un ordenador, un televisor último modelo, una vídeoconsola novedosa..., donde estos instrumentos son algo integrado y normalizado en el hogar. Se da por hecho que el momento y la sociedad actual nos exige tenerlo todo para estar al día, creando un sentimiento de necesidad; para los que tienen cultura es más sencillo evitar las trampas de la sociedad de consumo y la lógica de mercado, pero para los que tienen más carencias a este nivel esta estructura de vida se convierte en algo prioritario porque supone una condición de igualdad, al menos así se les hace sentir, por lo que ponen y anteponen todo su empeño en cubrir estos vacíos materiales para ser parte del "los otr@s".

Las nuevas tecnologías son un recurso atractivo para desarrollar acciones educativas con personas con un bagaje cultural bajo, para que puedan acceder a la educación y la cultura. Se trata de facilitar y acercar la información y la formación, así como las nuevas vías de comunicación de este siglo a todas las capas sociales, sin discriminación y de forma democrática.

Estos chicos están cerca y lejos al mismo tiempo de las nuevas tecnologías; cerca por su contacto con los más modernos medios, como ya he comentado, dentro de esa feroz campaña consumista que expone la sociedad, y lejos por su falta de capacidades y conocimientos para poder enfrentarse a tanto adelanto tecnológico de una forma enriquecedora y crítica; saben utilizar ordenadores para jugar a matar a puñetazos a la pandilla rival, simplemente apretando el botón del joystick; tienen mucha soltura para chatear y pasarse horas en la red ligando con chicas/os, tomando el pelo a unos y otros.

Realmente tienen mucho potencial y posibilidades, porque son chicos muy despiertos con los que no se ha intentado poner en juego prácticas educativas que relacionen sus vidas y sus intereses con el proceso de enseñanza-aprendizaje que podrían llegar a desarrollar.

Cuando me planteo en mi día a día con ellos utilizar el ordenador para buscar la información que necesitamos en un determinado programa informático que nos acerca a una enciclopedia, o cuando en lugar de hacerles escribir un dictado en un cuaderno les dejo al mando del teclado, o cuando buscamos en Internet un texto de su personaje favorito o de una afición que le mueve a aprender....; veo en sus caras interés, algo atractivo ha caído en sus manos y en sus mente. Este es el momento para aprovechar al máximo y ayudarles en sus procesos de aprendizaje, tanto de acceso a la cultura, como a las nuevas tecnologías como medio y herramienta para facilitar ese acceso. Pero para ello también es necesario disponer de medios y recursos para realizar las prácticas educativas, y por lo general con 
estos chicos se trabaja desde proyectos sociales, ya que han quedado excluidos del sistema educativo formal, al que tienen derecho como cualquier otro alumn@.

Sin embargo, desde nuestros proyectos no tenemos las mismas condiciones económicas ni recursos que nos permitan acercar a estos chicos a la tecnología actual, para poder estar en las mismas condiciones que los otros y hacer real el acceso a la formación, información y comunicación con el uso de las nuevas tecnologías. Es necesario que las administraciones y órganos competentes dediquen un esfuerzo a lo social, y piensen que el mundo que nos toca vivir con todas sus tecnologías también es el mundo de los que tienen menos, por lo que habría que dotar más y mejor aquellos centros o instituciones que puedan ser espacio de cultura y educación, tanto dentro del sistema formal dotando de recursos adecuados sin discriminación de zonas y colectivos, como los centros de educación no formal, insistiendo mucho en estos, ya que son más utilizados por las personas con mayor dificultad de integrarse en la sociedad. De no ser así estas tecnologías no harán sino crear otra barrera entre las personas atendiendo a su condición económica, cultural y social.

\section{Conclusiones}

Se pueden realizar las siguientes conclusiones y reflexiones de las que se irán configurando las líneas generales que creen el propio estilo y ritmo de enseñanza en este Proyecto (Taller Socioeducativo Ándara), apoyándose en la construcción de una nueva herramienta de trabajo (Ciberándara):

- Los chicos con los que se trabaja en este proyecto ponen esfuerzo cuando realizamos actividades vinculadas al ordenador y sus usos, esto hace que aumente su atención y el aprendizaje de lo que encuentran en su búsqueda. En una situación de aprendizaje tradicional (sentado en la silla realizando unas fichas, lectura....) el chico no aguantaría mucho tiempo, se levantaría, iría a otro espacio a ver que hacen sus compañeros, enredaría, hablaría de otras cosas, le costaría mucho mantener la atención; sin embargo cuando se sienta ante el ordenador es más fácil motivarle a realizar actividades que el educador ha preparado, aunque el contenido de lo que se le quiere enseñar sea el mismo que cuando estaba en la mesa; pero las formas no se parecen, el chico ni siquiera las relaciona; delante del ordenador se siente importante, incluso es consciente de que tiene que aprender a manejarlo porque oye hablar de la informática en la sociedad, de la utilidad de Internet, sabe que es algo que está a la orden del día.

- El/la educador/a tiene que ser para ellos un guía, orientar en los temas de búsqueda, sin evitar el que a veces sean ellos mismos quienes realicen las propuestas, pero con el objetivo de que lleguen a ser autónomos en el aprendizaje. Todo esto hace que la persona que educa con esta metodología deba poseer los conocimientos necesarios para ser un verdadero guía del proceso de enseñanza-aprendizaje; no es un mero transmisor de conocimientos que caen en un saco roto, sino un estímulo, un animador. Rompiendo con la figura del profesor tradicional, el educador del taller Ándara es cercano a los chicos, trabaja con una disciplina consciente pero desde un ambiente relajado, con pocos chicos, en espacios abiertos, en su propio entorno, con su grupo de iguales...

- Abandonar la rigidez de las instituciones educativas tradicionales, tender a la adaptación de todo aquello que sea necesario para llegar a estos chicos con éxito. Es interesante señalar un dato objetivo: en el 2004 hemos trabajado con chicos/as que habían abandonado el sistema escolar (expulsión, desmotivación, expedientes disciplinarios), se encontraban pasando todo el día en la calle, sin ningún interés, sin realizar ninguna actividad salvo rodearse de riesgos (consumos, robos,...). Desde febrero de 2004 hasta diciembre de 2004 han acudido al Taller ÁNDARA, han realizado actividades allí, han madrugado, han aprendido, han disminuido su tiempo de calle todas las mañanas de lunes a viernes, incluso en la temporada de verano, ya que las vacaciones en este proyecto no son como las escolares; esta realidad hace pensar en que sí hay medidas posibles para trabajar con ellos, pero que rompen el modelo tradicional de escuela; no son chicos imposibles, no son seres peligrosos sino en peligro, por lo tanto el mundo de la educación debería parar y pensar, analizar lo que realmente pasa en la calle, en las vidas de estos chicos. 
Acción-reflexión-acción, método participativo, rescatar los principios pedagógicos que ya en otros tiempos se encaminaron a este estilo de educación, con la novedad de introducir las nuevas tecnologías para preparar la educación y la sociedad del presente y del futuro.

- Planteamiento abierto hacia las TIC. No un discurso de rechazo, pero siempre con un espíritu crítico y acorde con la filosofía social que está detrás de nuestro trabajo. La educación en general y la educación social en particular ha de buscar un cambio en las estructuras sociales, no debe conformarse con la actividad del día a día; este será uno de los pasos para poder caminar hacia la transformación social.

- Cambio de espacio (no el aula convencional), con el objetivo de llamar la atención de los chicos, sorprenderles cada día con las actividades a realizar, que no se aburran, no ser rígidos en la transmisión de contenidos, no forzar programaciones por el simple hecho de querer cumplirlas en un plazo determinado, partir de la situación real y del estado de los chicos y de la dinámica del taller.

- Formación para la vida actual, ofrecer la posibilidad de acceder a las nuevas tecnologías (potenciar un acceso y participación democráticas). Dar facilidad para aprender en espacios que acerquen la cultura audiovisual e informática, y para desarrollar herramientas que cualifiquen a los chicos para un futuro profesional, reduciendo las dificultades que este colectivo tiene en la inserción laboral.

- No podemos olvidar que la nueva tecnología está a la orden del día para muchos, pero que muchos otros se encuentran aún en plena alfabetización, o inmersos en un fuerte retraso y vacío cultural; hay que evitar posturas que limiten los nuevos medios a los que tienen información y formación, y hacer que tengan un uso real entre los que no han tenido esas posibilidades. Nadie puede negar que la tecnología resulta atractiva, y puede en muchas ocasiones conseguir más resultados que otros medios de transmitir conocimientos. Por este motivo puede convertirse en una ayuda muy positiva para el educador o profesor en el proceso de enseñanza-aprendizaje (sin olvidar que esta figura como guía es fundamental, para conducir estas prácticas por el camino adecuado).

Para terminar, es importante mencionar una cuestión de gran importancia; los sistemas educativos son capaces de generar inclusión o exclusión escolar, la cual conlleva inclusión o exclusión social. No es que la primera sea exclusivamente la causa de la segunda, pero acentúa fuertemente ese proceso de construcción social.

El colectivo al que se ha hecho mención a lo largo de esta experiencia pertenece a zonas de exclusión social rodeadas de factores de riesgo (familia, entorno, uso del tiempo libre marcado por la estancia en la calle, consumo y dependencia de sustancias tóxicas, desinterés por el aprendizaje, inexistencia de perspectivas de futuro, delitos y trapicheos...). Ante este panorama la vida de un niño/a en estas condiciones puede estar marcada o condicionada dependiendo de que su paso por el sistema escolar haya sido integrador o exclusor; cada vez son más los chicos de estas zonas sociales que abandonan los centros educativos a los 12-13 años, coincidiendo con el paso a la educación secundaria; su motivación, desinterés y absentismo escolar ya venían de años previos, pero en estas edades se produce la ruptura definitiva con ese medio, en el que no se integran ni hay medidas suficientes ni adecuadas para que esto sea posible. El hecho de que con esta edad acaben en la calle hace aumentar su proceso exclusor, faltados de las capacidades mínimas necesarias que les impedirán poder crear un proyecto de vida, de futuro.

Es así como, aún viniendo de entornos de exclusión y marginación, mediante experiencias como las que se ofrecen en este artículo esos niños/as podrán aprender y construir otro futuro para sus vidas, por muchas barreras que esta les vaya presentando en el camino, pero lo que hayan aprendido quedará ahí para ayudarles; será la riqueza que consigan llevarse consigo mismos. 
para la Formación Profesional Ocupacional. Málaga: Facep

AREA MOREIRA, M. "Nuevas Tecnologías, desigualdad y educación en las sociedades de la información”.www.ieev.uma.es/edutec97/edu97_c4/2-4-14.htm

AREA MOREIRA, M. (1997): "Futuro imperfecto. Nuevas Tecnologías y desigualdades educativas", en http://www.ull.es/publicaciones/tecinfedu/index.html

CABERO, J.: "El ciberespacio: el no lugar como lugar educativo", en SALINAS, J. et al. (eds.): Redes de Comunicación, redes de aprendizaje. Palma: Universidad de las Islas Baleares; 77-90

CABERO, J. (Coord) (2000) Las nuevas tecnologías para la mejora educativa. Sevilla: Kronos

CABERO, J. et al. (1996) Medios de comunicación, recursos y materiales para la mejora educativa II. Sevilla: CMIDE

CASTELLS, M; FLECHA, R.; FREIRE, P., GIROUX,D. y WILLIS, P. (1997): Nuevas Perspectivas Críticas en Educación. Madrid: Paidós.

ILLICH, I. (1974): La sociedad desescolarizada. Barcelona: Barral Editores

MONEREO FONT, C. y POZO MUNICIO, J. I. (2001) “¿En qué siglo vive la escuela?” Cuadernos de Pedagogía, 298, enero 2001

PUIG DE LA BELLACASA, R.: "Las sociedades de la información ante los procesos de exclusión social", en Telos. Cuadernos de Comunicación, Tecnología y Sociedad, versión electrónica http://www.fundesco.es/publica/telos.html.

(C) Ediciones Universidad de Salamanca 